\title{
A RESULT ON BEST APPROXIMATION
}

\author{
NASEER SHAHZAD
}

\begin{abstract}
Using a common fixed point theorem for noncommuting mappings of Pant [4], we improve and extend a result of Sahab, Khan and Sessa [5] on best approximation.
\end{abstract}

Throughout this paper, $E$ denotes a normed space. A subset $C$ of $E$ is said to be starshaped with respect to a point $p \in C$ if, for each $x \in C$, the segment joining $x$ to $p$ is contained in $C$ (that is , $\lambda x+(1-\lambda) p \in C$ for each $x \in C$ and real $\lambda$ with $0 \leq \lambda \leq 1$ ). $C \subset E$ is said to be starshaped if it is starshaped with respect to one of its elemnts. A convex set obviously starshaped. A mapping $T: E \rightarrow E$ is nonexpansive on $E$ (resp. on a. subset $C$ of $E$ ) if $\|T x-T y\| \leq\|x-y\|$ for all $x, y \in E$ (resp. for all $x, y \in C$ ). The set of fixed points of $T$ in $E$ is denoted by $F(T)$. Suppose $\hat{x} \in E$. An element $y \in C$ is called an element of best approximation of $\hat{x}$ if we have $\|\hat{x}-y\|=\inf _{z \in C}\|\hat{x}-z\|$. We will denote by $P_{c}(\hat{x})$ the set of all such elements $y$. We denote the boundary of $C$ by $\partial C$.

In 1969, Brosowski [1] obtained the following result which generalizes a theorem of Meinardus [3]:

Theorem 1. Let $T: E \rightarrow E$ be a linear and nonexpansive operator on $E$. Let $C$ be a $T$-invariant subset of $E$ and let $\hat{x} \in F(T)$. If $P_{c}(\hat{x})$ is nonempty, compact, and convex then $P_{c}(\hat{x}) \cap F(T) \neq \phi$.

In 1979, Singh [6] observed that the linearity of the operator $T$ and the convexity of $P_{c}(\hat{x})$ in Theorem 1 can be relaxed and gave the following extension of it.

Theorem 2. Let $T: E \rightarrow E$ be a nonexpansive operator on $E$. Let $C$ be a $T$ invariant subset of $E$ and let $\hat{x} \in F(T)$. If $P_{c}(\hat{x})$ is nonempty, compact, and starshaped, then $P_{c}(\hat{x}) \cap F(T) \neq \phi$.

Singh [7] showed that if $D^{\prime}=P_{c}(\hat{x}) \cup\{\hat{x}\}$, then Theorem 2 remains valid for $T$ satisfying the condition of nonexpansiveness only on $D^{\prime}$. Recently, Sahab, Khan and Sessa [5] generalized Theorem 2 with the following result.

Theorem 3. Let $T, I: E \rightarrow E$ be operators, $C$ be a subset of $E$ such that $T: \partial C \rightarrow$ $C$, and $\hat{x} \in F(T) \cap F(I)$. Further $T$ and I satisfy

$$
\|T x-T y\| \leq\|I x-I y\|
$$

for all $x, y \in D^{\prime}=P_{c}(\hat{x}) \cup\{\hat{x}\}$ and let $I$ be linear, continuous on $P_{c}(\hat{x})$, and IT $x=T I x$ for all $x \in P_{c}(\hat{x})$. If $P_{c}(\hat{x})$ is nonempty, compact and starshaped with respect to a point $p \in F(I)$ and if $I\left(P_{c}(\hat{x})\right)=P_{c}(\hat{x})$, then $P_{c}(\hat{x}) \cap F(T) \cap F(I) \neq \phi$.

Received December 15, 1997. 
Remark 4. In Theorem 3, the hypothesis that $I$ is continuous implies that $T$ is continuous. In [5] Sahab, Khan and Sessa used the continuity of both $T$ and $I$.

We need the following preliminary definitions and results.

Let $(X, d)$ be a metric space and let $T$ and $I$ be self-mappings of $X$. The mappings $T$ and $I$ will be called $R$-weakly commuting on $X$, provided there exists some positive real number $R$ such that

$$
d(T I x, I T x) \leq R d(T x, I x)
$$

for each $x \in X . T$ and $I$ will be called $R$-weakly commuting and a point $x$ if $d(T I x, I T x) \leq R d(T x, I x)$ for some $R>0$. Obviously, weak commutativity implies $R$-weak commutativity. However, $R$-weak commutativity implies weak commutativity only when $R \leq 1$. For details of the above see Pant [4].

The following is a consequence of Theorem 1 of Pant [4].

Theorem 5. Let $(X, d)$ be a complete metric space and let $T, I: X \rightarrow X$ be $R$-weakly commuting mappings such that $T(X) \subseteq I(X)$, and $d(T x, T y)<d(I x, I y)$ whenever $I x \neq I y$. If either $T$ or $I$ is continuous, then $F(T) \cap F(I)$ is singleton.

Let us continue this paper by observing that even if in Theorem 3 the conditions of continuity and commutativity of operators are somewhat relaxed, the assertion of Theorem 3 remains valid. Thus we get the following interesting result, which is new in the sense that, unlike other authors (see Remark 4), we do not require both $T$ and $I$ to be continuous.

Theorem 6. Let $T, I: E \rightarrow E$ be operators, $C$ be a subset of $E$ such that $T: \partial C \rightarrow$ $C$, and $\hat{x} \in F(T) \cap F(I)$. Further $T$ and $I$ satisfy (1) on $D^{\prime}=P_{c}(\hat{x}) \cup\{\hat{x}\}$ and let $I$ be linear on $P_{c}(\hat{x})$ and $T, I$ be $R$-weakly commuting on $P_{c}(\hat{x})$. If $P_{c}(\hat{x})$ is nonempty, compact and starshaped with respect ot $p \in F(I)$, if $I\left(P_{c}(\hat{x})\right)=P_{c}(\hat{x})$, and if either $T$ or $I$ is continuous, then $P_{c}(\hat{x}) \cap F(T) \cap F(I) \neq \phi$.

Proof. First, we show that $T: P_{c}(\hat{x}) \rightarrow P_{c}(\hat{x})$. Let $y \in P_{c}(\hat{x})$ and hence $I y \in P_{c}(\hat{x})$ since $I\left(P_{c}(\hat{x})\right)=P_{c}(\hat{x})$. Then $y \in \partial C$ (see Hicks and Humphries [2]) implying that $T y \in C$, since $T: \partial C \rightarrow C$. It follows from (1) that

$$
\|T y-\hat{x}\|=\|T y-T \hat{x}\| \leq\|I y-I \hat{x}\|=\|I y-\hat{x}\|
$$

and therefore $T y \in P_{c}(\hat{x})$.

Let us define a sequence of maps $T_{n}$ :

$$
T_{n} x=\left(1-k_{n}\right) p+k_{n} T x
$$

where $k_{n}$ is a fixed sequence of positive numbers less than 1 and converging to 1 . Each $T_{n}$ maps $P_{c}(\hat{x})$ into itself because $T: P_{c}(\hat{x}) \rightarrow P_{c}(\hat{x})$ and $P_{c}(\hat{x})$ is starshaped with respect to $p$. Since $I$ is linear and $R$-weakly commutes with $T$ on $P_{c}(\hat{x})$, we have

$$
\begin{aligned}
& T_{n} I x=\left(1-k_{n}\right) I p+k_{n} T I x \\
& I T_{n} x=\left(1-k_{n}\right) I p+k_{n} I T x
\end{aligned}
$$


and

$$
\begin{aligned}
\left\|T_{n} I x-I T_{n} x\right\| & =k_{n}\|T I x-I T x\| \\
& \leq k_{n} R\|T x-I x\| \\
& <R\|T x-I x\|
\end{aligned}
$$

for all $x \in P_{c}(\hat{x})$. Thus $T_{n}$ and $I$ are $R$-weakly commuting on $P_{c}(\hat{x})$ for each $n$ and $T_{n}\left(P_{c}(\hat{x})\right) \subseteq I\left(P_{c}(\hat{x})\right)$. Also, we have

$$
\left\|T_{n} x-T_{n} y\right\|=k_{n}\|T x-T y\| \leq k_{n}\|I x-I y\|<\|I x-I y\|
$$

whenever $I x \neq I y$.

Since either $T$ or $I$ is continuous, according to Theorem $5 F\left(T_{n}\right) \cap F(I)=\left\{x_{n}\right\}$ for each $n$. Since $P_{c}(\hat{x})$ is compact, $\left\{x_{n}\right\}$ has a subsequence $\left\{X_{n_{i}}\right\} \rightarrow z$ (say) in $P_{c}(\hat{x})$.

Let us suppose that the mapping $T$ is continuous. Since

$$
x_{n_{i}}=T_{n_{i}} x_{n_{i}}=\left(1-k_{n_{i}}\right) p+k_{n_{i}} T x_{n_{i}}
$$

we have, as $i \rightarrow \infty$, that $z=T z$, that is $z \in P_{c}(\hat{x}) \cap F(T)$. Since $T_{n}$ and $I$ are $R$-weakly commuting, we have that

$$
\left\|T_{n_{i}} I x_{n_{i}}-I T_{n_{i}} x_{n_{i}}\right\| \leq R\left\|T_{n_{i}} x_{n_{i}}-I x_{n_{i}}\right\|
$$

On letting $i \rightarrow \infty$, the above inequality yields $I T_{n_{i}} x_{n_{i}} \rightarrow T z=z$ since $T_{n_{i}} x_{n_{i}}=$ $I x_{n_{i}}=x_{n_{i}}$. Since $T\left(P_{c}(\hat{x})\right) \subseteq P_{c}(\hat{x})=I\left(P_{c}(\hat{x})\right)$ it follows from $z=T z$ that there exists $z_{1} \in P_{c}(\hat{x})$ such that $z=T z=I z_{1}$. Now

$$
\left\|T T_{n_{i}} x_{n_{i}}-T z_{1}\right\| \leq\left\|I T_{n_{i}} x_{n_{i}}-I z_{1}\right\| .
$$

This inequality on letting $i \rightarrow \infty$ implies that $T z=T z_{1}$ since $I T_{n_{i}} x_{n_{i}} \rightarrow T z$ and $T z=T z_{1}$.

Thus $z=T z=T z_{1}=I z_{1}$. This in turn implies that

$$
\|T z-I z\|=\left\|T I z_{1}-I T z_{1}\right\| \leq R\left\|T z_{1}-I z_{1}\right\|=0
$$

that is, $z=T z=I z$ and hence

$$
P_{c}(\hat{x}) \cap F(T) \cap F(I) \neq \phi .
$$

The same conclusion is found when $I$ is assumed to be continuous since continuity of $I$ implies continuity of $T$.

We have improved and extended Theorem $3([5])$ for noncommuting maps. 


\section{References}

[1] B. Brosowski, "Fixpunktsatze in der approximations-theorie," Mathematica (Cluj), 11(1969), 195-220.

[2] T. L. Hicks and M. D. Humphries, "A note on fixed point theorems," J. Approx. Theory, 34(1982), 221-225.

[3] G. Meinardus, "Invarianz bei linearen approximationen," Arch. Rational Mech. Anal., 14(1963), 301-303.

[4] R. P. Pant, "Common fixed points of noncommuting mapping," J. Math. Anal. Appl., 188(1994), 436-440.

[5] S. A. Sahab, M. S. Khan and S. Sessa, "A result in best approximation theory," J. Approx. Theory, 55(1988), 349-351.

[6] S. P. Singh, "An application of a fixed point theorem to approximation theory," J. Approx. Theory, 25(1979), 89-90.

[7] S. P. Singh, "Applications of fixed point theorems in approximation theory," in Applied Nonlinear Analysis, (V. Lakshmikantham, Ed.), Academic Press, New York, 389-394, 1979.

Department of Mathematics, Quaid-i-Azam University, Islamabad-Pakistan. 